\section{Commentary: Better genetics, less pathology}

\author{
Ruggero De Paulis, MD
}

It is unsurprising that in the recent article by Tamer and colleagues, ${ }^{1}$ coming from a well-known center of aortic valve repair, the number of patients undergoing this type of surgery was rather small. Indeed, the authors report only 127 cases of isolated tricuspid valve repair (TAV) over a 24year period, for an average of approximately 5 cases per year. If we consider that 21 patients underwent reoperation after a Ross procedure or aortic valve-sparing surgery, this number is even smaller. Although the number of cases may appear too small to enable meaningful conclusions, it represents the true incidence of this pathology in an adult population, and it is remarkable that the authors collected and treated this number of patients.

The presence of severe aortic regurgitation necessitating surgery in the absence of a root or an ascending aneurysm is very rare in TAV. In such cases, the lesion responsible for severe aortic regurgitation is usually found in the annular dilatation, the leaflet prolapse, or both. These are indeed the types of lesions common in the bicuspid aortic valve configuration, where the genetic alteration usually hits the annulus with evident annuloectasia and consequently the conjoined cusp with a large prolapsing free margin. On the other hand, a tricuspid valve configuration has no clear underlying genetic alterations that justify or explain the presence of specific anatomic lesions. In fact, the authors included only 127 patients in their cohort, having excluded all patients with rheumatic disease, connective disorders, endocarditis, dissection, or aortic dilatation. The curative surgical interventions were aimed mainly at annular

\footnotetext{
From the Cardiac Surgery Department, European Hospital, UniCamillus-Saint Camillus International University of Health Sciences, Rome, Italy.

Disclosures: The author reported no conflicts of interest.

The Journal policy requires editors and reviewers to disclose conflicts of interest and to decline handling or reviewing manuscripts for which they may have a conflict of interest. The editors and reviewers of this article have no conflicts of interest.

Received for publication April 19, 2021; revisions received April 19, 2021; accepted for publication April 20, 2021; available ahead of print April 29, 2021.

Address for reprints: Ruggero De Paulis, MD, Cardiac Surgery Department, European Hospital, via Portuense 700, 00151 Rome, Italy (E-mail: rdepaulis58@ gmail.com or ruggero.depaulis@unicamillus.org).

J Thorac Cardiovasc Surg 2023; 165:1007-8

$0022-5223 / \$ 36.00$

Copyright (c) 2021 by The American Association for Thoracic Surgery

https://doi.org/10.1016/j.jtcvs.2021.04.054
}

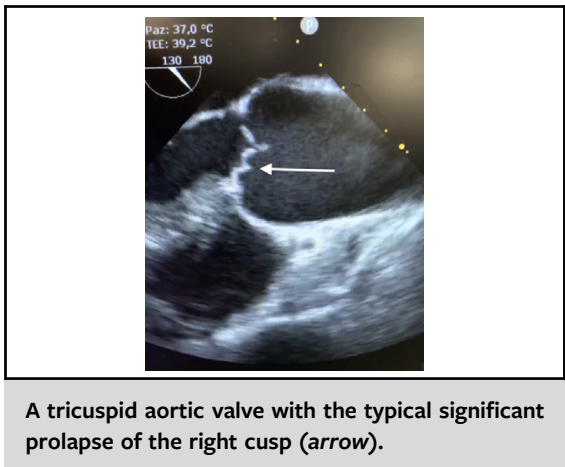

CENTRAL MESSAGE

Isolated tricuspid valve insufficiency is a rare finding that is less linked to genetic anomalies. A proper understanding of the mechanism is needed to precisely tailor the surgical treatment.

reduction (99\% of annuloplasties) associated with cusp repair $(92 \%)$. However, even though annuloplasty was performed in the great majority of cases by means of a subcommissural annuloplasty (or Cabrol stitch), generally considered a suboptimal and less durable form of annular reduction by the same group, ${ }^{2}$ the results indicate that the annuloplasty technique did not influence the results. Medium- and long-term failures were ascribed mainly to the severity of cusp pathology. These simple findings seem to indicate how annuloplasty plays a supporting role in cusp repair in cases of TAV but a major curative role in bicuspid aortic valve, where the genetic alteration in the annulus is more evident and severe. The treatment is similar, but the underlying pathology differs.

Another important aspect to consider in this context is the cusp responsible for the prolapse and the aortic regurgitation. For whatever reason, the most frequently affected leaflet is the right cusp, which is more frequently and more easily addressed. When more than one cusp is involved or when the left cusp is involved (as described by Tamer and colleague), a caution bell should ring. In such cases, it is relatively more difficult to achieve optimal leaflet coaptation.

In conclusion, the presence of severe aortic valve insufficiency necessitating surgery is a rare finding in a tricuspid valve configuration. Be aware that in isolated TAV regurgitation, pathology in the skeleton of the root 
is not always the main problem, and a trickier and more difficult gesture on the aortic leaflet is to be expected. A lesion of the left cusp is probably a sign of the need for more complex and less durable repair. Avoiding cusp reconstruction by means of pericardial patch will avoid early failure of the repair.

\section{References}

1. Tamer S, Mastrobuoni S, Vancraeynest D, Lemaire G, Navarra E, El Khoury G, et al. Late results of aortic valve repair for isolated severe aortic regurgitation. $J$ Thorac Cardiovasc Surg. 2023;165:995-1006.e3.

2. de Kerchove L, Mastrobuoni S, Boodhwani M, Astarci P, Rubay J, Poncelet A, et al. The role of annular dimension and annuloplasty in tricuspid aortic valve repair. Eur J Thorac Cardiovasc Surg. 2006;49:428-37.
See Article page 995.

\section{Commentary: Repair of the tricuspid aortic valve: Simplicity is the ultimate sophistication}

\author{
Stephanie L. Mick, MD, and \\ Mario Gaudino, MD, PhD, MSCE
}

In this edition of the Journal, Tamer and colleagues ${ }^{1}$ analyzed the long-term outcomes associated with repair of a fairly infrequent aortic valve pathology, isolated tricuspid aortic valve (TAV) regurgitation (AR). While there are plentiful data showing favorable durability of repair for aortic valves with bicuspid morphology (bicuspid aortic valve [BAV]) and further, that circumferential annuloplasty techniques (ring or reimplantation) improve the stability of repair, ${ }^{2-8}$ similar data for TAV have been lacking. ${ }^{9}$

The authors aim to fill this gap in this single-center, retrospective analysis of 127 patients over 23 years who underwent aortic valve repair in the setting of TAV without aortic root or ascending aorta dilation $(\geq 45 \mathrm{~mm})$, connective tissue disease, endocarditis, or dissection. For the first decade in the experience, Cabrol annuloplasty sutures were employed for annular stabilization. After 2009, external ring annuloplasty was added to repairs in patients with normal root dimensions and valve reimplantation added when

From the Department of Cardiothoracic Surgery, Weill Cornell Medicine, New York, NY.

Disclosures: The authors reported no conflicts of interest.

The Journal policy requires editors and reviewers to disclose conflicts of interest and to decline handling or reviewing manuscripts for which they may have a conflict of interest. The editors and reviewers of this article have no conflicts of interest.

Received for publication April 21, 2021; revisions received April 21, 2021; accepted for publication April 22, 2021; available ahead of print April 30, 2021.

Address for reprints: Mario Gaudino, MD, PhD, MSCE, Department of Cardiothoracic Surgery, Weill Cornell Medicine, 525 E 68th St, New York, NY 10065 (E-mail: mfg9004@med.cornell.edu).

J Thorac Cardiovasc Surg 2023;165:1008-9

$0022-5223 / \$ 36.00$

Copyright (c) 2021 by The American Association for Thoracic Surgery

https://doi.org/10.1016/j.jtcvs.2021.04.071
Check for updates

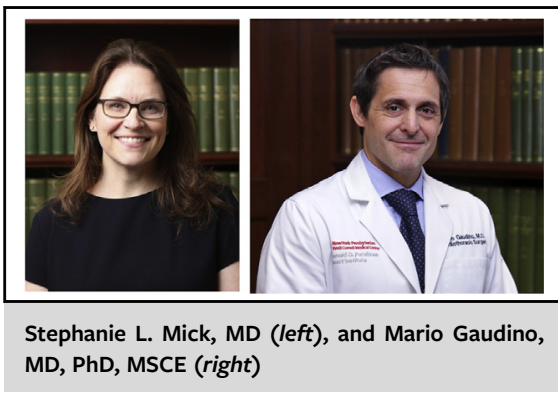

CENTRAL MESSAGE

Durability of repair of tricuspid

aortic valve regurgitation may

decline as overall complexity in-

creases, and there is not yet suf-

ficient evidence to establish the

most effective annuloplasty type.

root diameter exceeded $40 \mathrm{~mm}$. Cusp repair techniques included cusp plication, cusp resuspension, or pericardial patch repairs in the case of large/ruptured fenestrations, perforations, or retractions.

Cusp repair was performed in 117 (92\%) and annuloplasty in $126(99 \%)$. There were no in hospital mortalities, 10 - and 14-year survival was $81 \pm 5 \%$ and $71 \pm 6 \%$, and freedom from reoperation was $80 \pm 5 \%$ and $73 \pm 6 \%$, respectively. Freedom from recurrent severe AR was $73 \pm 5 \%$ and $66 \pm 7 \%$ at 10 and 12 years. Cabrol annuloplasties made up a far larger proportion (73\%) of this population than circumferential techniques (reimplantation or ring annuloplasties).

No difference in reoperation or AR recurrence was shown between annuloplasty techniques, which is different than in BAV, where circumferential techniques have been associated with improved durability. ${ }^{4,5,10}$ The authors do 